\title{
The injector microtron for the TEUFEL infrared laser
}

\author{
J.I.M. Botman, G.A. Webers, J.L. Delhez, C.J. Timmermans, M.E.H.J. Theeuwen, \\ W.J.G.M. Kleeven and H.L. Hagedoorn \\ Eindhocen Unicersity of Technology, Cyclotron Laboratory, P.O. Box 513, 5600 MB Eindhoien, Netherlands
}

\author{
G.J. Ernst, J.W.J. Verschuur and W.J. Witteman \\ Twente Unicersity, P.O. Box 217, 7500 AE Enschede, Netherlands
}

\begin{abstract}
Progress is reported on a $25 \mathrm{MeV}$ injector racetrack microtron for a $10 \mu \mathrm{m}$ radiation free electron laser (TEUFEL project). The accelerator exhibits transverse focusing in $180^{\circ}$ inhomogeneous two-sector dipole magnets which are slightly rotated with respect to each other in the bending plane. This provides closed orbits, isochronism and a large transverse acceptance. Details on this unconventional microtron focusing system will be given. An analytical treatment, based on conformal mapping, of the field near pole boundaries and at the hill-valley boundaries in the microtron dipole is compared with Poisson calculated results and with field measurements. The design of a model accelerating cavity is presented together with field measurements based on the perturbation ball method.
\end{abstract}

\section{Introduction}

In the second phase of the TEUFEL project [1] a racetrack microtron (RTM) will accelerate 50-100 A pulses from a $6 \mathrm{MeV}$ photocathode injector linac up to an energy of $25 \mathrm{MeV}$, to provide $10 \mu \mathrm{m}$ radiation in an undulator with a periodicity distance of $2.5 \mathrm{~cm}$ [2]. Studies are progressing on the focusing properties of the RTM, required for the acceleration of the intense beam. The RTM has an average magnetic field in the bending magnets of $0.19 \mathrm{~T}$ and a magnet separation of $0.8 \mathrm{~m}$; it has 9 orbits with an acceleration voltage of 2.2 MV at $1.3 \mathrm{GHz}$

In this paper we discuss several developments of the RTM design, such as those on the focusing features, and on the model cavity construction and measurements.

\section{Two sector magnet}

For obtaining proper transverse beam focusing, a useful combined function RTM magnet design was first introduced by Froelich et al. [3]. The bending magnets were not homogeneous but consisted of three distinct triangular field regions, still yielding a $180^{\circ}$ bend for all energies, but also creating two quadrupoles, one at each boundary between adjacent field regions (edge focusing). Calculations using this layout showed that the focusing properties obtained were satisfactory yet very sensitive to small changes in design parameters [4].

We have therefore turned to another, less conventionai layout, in which the magnets consist of just two field regions ("two-sector magnet"), yielding a single quadrupole per magnet. For obtaining a $180^{\circ}$ bend, the magnets have to be rotated in their median plane through an angle $\tau$ in such a way that the outgoing beam is parallel to the incoming beam, despite two different field levels in the magnet (see fig. 1). This design turns out to have much better specifications: the

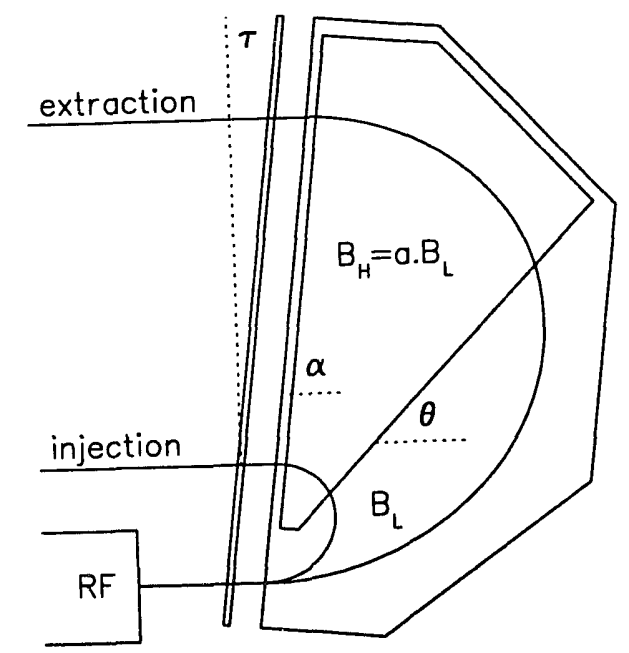

Fig. 1. Schematic depiction of the right-hand two-sector magnet; only two orbits are shown for clarity. 
microtron acceptance is larger and also considerably less sensitive to (even large) changes in parameters.

Thanks to the magnet rotation and the field edge within the magnet, the beam experiences two weak, vertically focusing quadrupole lenses and one weak, vertically defocusing lens per pass through the magnet.

The two field levels in the magnet are indicated as $B_{\mathrm{L}}$ and $B_{\mathrm{H}}$. The angles $\theta$ (angle of $\mathrm{L} / \mathrm{H}$ boundary) and $\alpha=\pi / 2-\tau$ (magnet angle) are defined with respect to the direction of the incoming beam. We write

$B_{\mathrm{H}}=a B_{\mathrm{L}} ; \quad a \geq 1$

and find

$\tan (\alpha)=\frac{a+1}{(a-1) \sin (2 \theta)}-\frac{1}{\tan (2 \theta)}$.

As can be seen, $a$ and $\theta$ are the degrees of freedom. The angle $\alpha$ is fixed for each choice of these two parameters. Also, one easily sees that for any choice of the set $(a, \theta)$, the bending length through the magnet depends linearly on the radius of curvature $\rho$ of a particle with given energy. Consequently, isochronism (i.e. the orbit lengths are integer multiples of the accelerating structure wavelength for all energies required) can always be reached, merely by adjusting the field strength of the magnet: if a perfectly homogeneous magnet with induction $\langle B\rangle$ fulfilles the isochronism condition (for given drift length), then the twosector layout must have

$B_{\mathrm{L}}=\left\{\left(1-\frac{1}{a}\right) \frac{2 \theta+\sin (2 \theta)}{\pi}+\frac{1}{a}\right\}\langle B\rangle$

and $B_{H}$ according to eq. (1). The focal lengths of the three quadrupole lenses are:

$f_{\text {in }}=-\rho_{\mathbf{H}} / \tan (\tau)$,

$f_{\mathrm{L} / \mathrm{H}}=+\rho_{\mathrm{H}} \tan (\theta) /(a-1)$,

$f_{\text {out }}=+\rho_{\mathrm{H}} /(a \tan (\tau))$, the first being vertically defocusing, the latter two vertically focusing.

Using matrix theory, one can "track" particles from injection to extraction through a system of drift spaces, dipoles and quadrupoles. Doing so, one can calculate the beam acceptance of the microtron by transforming all boundaries back to the point of injection (in phase space), together building up a "transverse separatrix". The area inside the separatrix defines the acceptance of the machine. If the first order transport matrix for a transverse deviation $y$ (divergence $y^{\prime}$ ) from injection to longitudinal orbit position $s$ is given by

$$
\begin{aligned}
& \left(\begin{array}{c}
y(s) \\
y^{\prime}(s)
\end{array}\right)=\left(\begin{array}{cc}
a_{0}(s) & a_{1}(s) \\
\mathrm{d} a_{0} / \mathrm{d} s & \mathrm{~d} a_{1} / \mathrm{d} s
\end{array}\right)\left(\begin{array}{c}
y(0) \\
y^{\prime}(0)
\end{array}\right) ; \\
& y \in\{r, z\}
\end{aligned}
$$

the acceptance $A_{v}$ (full area in $\mathrm{m} \mathrm{rad}$ ) can be written as

$$
\begin{aligned}
A_{y}=\frac{G_{0}^{2}}{2[\mathrm{~m}]} \int_{0}^{2 \pi}\left\{\max _{s} \mid a_{0}(s) \cos (\phi)\right. \\
\left.+\frac{a_{1}(s)}{[\mathrm{m}]} \sin (\phi) \mid\right\}^{-2} \mathrm{~d} \phi,
\end{aligned}
$$

with $G_{0}$ being the half-aperture of the machine (assumed constant here). The acceptance is a good measure for focusing quality. For the results presented in this article, the particles are tracked through calculated and measured field maps. The matrix elements $a_{0}(s)$ and $a_{1}(s)$ are derived from the field gradient rectangular to the orbit. For generating numerical field maps, use can be made of fringe field calculations [5] (see also next section) as well as of measurements. This kind of tracking also provides us with useful data about orbit lengths and closed orbit distortions.

By calculating the radial and vertical acceptance for many combinations of the parameters $(a, \theta)$, one can get an idea where the optimum is located. Our defini-
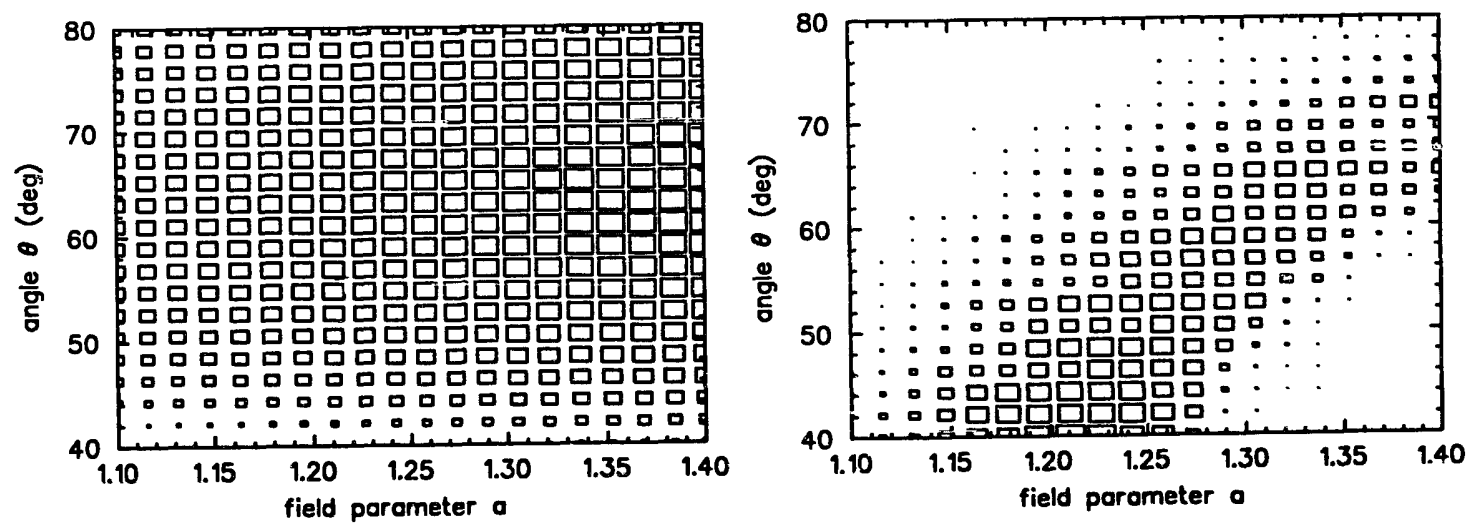

Fig. 2. Density plot of radial (left) and vertical (right) acceptance as a function of $a$ and $\theta$ for the two-sector magnet. 

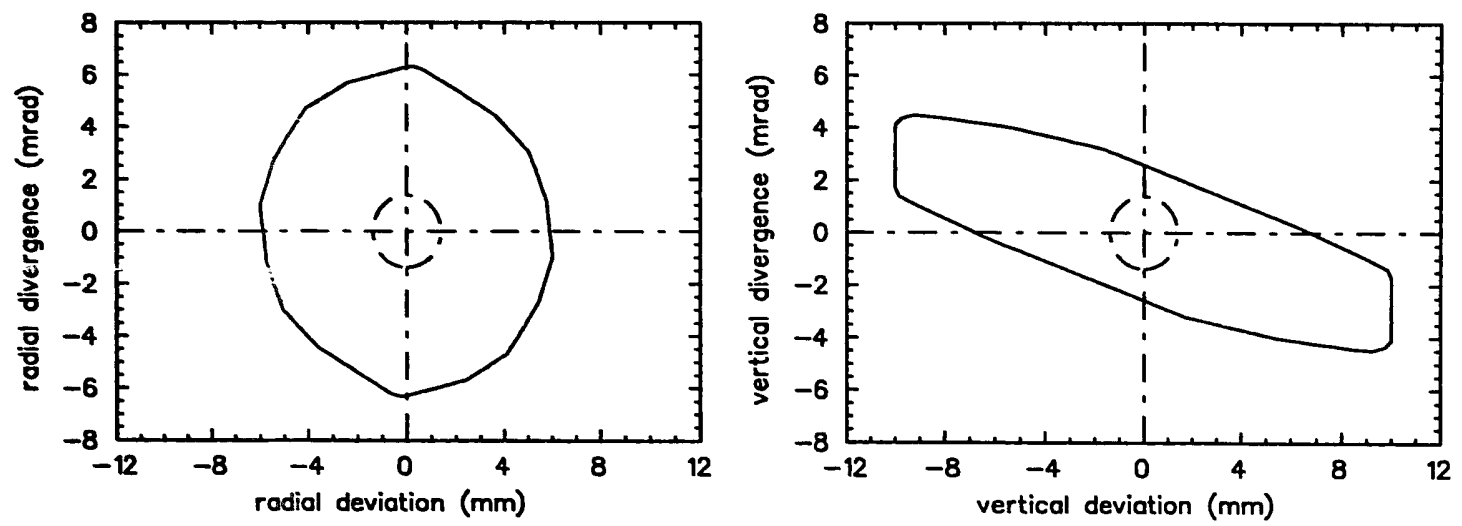

Fig. 3. The radial and vertical separatrix for the optimum two-sector magnet configuration. The dashed circle inside each separatrix is the emittance of the beam at injection (assumed circular).

tion of the optimum $(\hat{a}, \hat{\theta})$ is that the smaller one of the radial and vertical acceptance at $(\hat{\boldsymbol{a}}, \hat{\theta})$ must be larger than the smaller one at any other $(a, \theta)$.

Fig. 2 shows density plots of the radial and vertical acceptance of the two-sector RTM, based on calculations of $\mathbf{4 0 0}$ configurations in the plotted region for the current 6-25 MeV RTM. The radial acceptance is large in the entire region, whereas the vertical acceptance remains large over a wide range of $(a, \theta)$. The optimum (as defined above) is found at $\hat{a}=1.25, \hat{\theta}=$ $50^{\circ}$ and the acceptance values are $A_{r}=110 \mathrm{~mm} \mathrm{mrad}$, $A_{z}=90 \mathrm{~mm} \mathrm{mrad}$ (for $G_{0}=10 \mathrm{~mm}$ ). Both of these values are much larger than the requested $6 \mathrm{~mm}$ mrad [2]. Additionally, one can change the parameters freely from $\left(a=1.2, \theta=50^{\circ}\right)$ up to $\left(a=1.3, \theta=60^{\circ}\right)$ without significant change of acceptance values. This makes the optimum rather insensitive to design imperfections.

Finally, fig. 3 shows the radial and vertical separatrices (drawn line) at $\left(\hat{a}=1.25, \hat{\theta}=50^{\circ}\right)$. The radial separatrix is almost circular, the vertical separatrix is slightly stretched along the displacement axis. The dotted circle inside each separatrix indicates the emittance of the beam at injection (assumed circular).

\section{Fringe field properties}

In order to study the transverse and longitudinal stability in the microtron, a first order matrix description is used. In this theory we used a hard edge description of the magnetic field. In order to describe the finite extent of the fringe fields, we use the quantities $E F B$ and $I_{2}$. The EFB (effective field boundary) represents the conservation of bending angle and $I_{2}$ represents the defocusing force in the vertical direction of the fringe field. This implies that the entrance angle $\alpha$ in the expression for the edge focusing strength $\tan (\alpha) / \rho$ is replaced by an effective angle $\alpha$, given by [6]:

$\tan \alpha_{\mathrm{v}}=\tan \alpha-\frac{g I_{2}\left(1+\sin ^{2} \alpha\right)}{\rho \cos ^{3} \alpha}$.

$I_{2}$ depends on the fringe field in the median plane. It is our aim, to determine the fringe field quantities $E F B$ and $I_{2}$ as a function of the geometry.

\section{Conformal mapping}

In order to calculate the magnetic field distribution in the median plane we used the mathematical tool of conformal mapping (CM). Before setting up this analytical theory, we make some simplifications: first, we consider only regions with no current enclosed. This implies that the magnetic field is expressed in terms of a scalar potential, which is a solution of the Laplace equation. Secondly, we do not include saturation effects, so the potential along the pole boundary is constant. Because of r.idplane symmetry the potential in the median plane :s also constant. So the problem of solving the magnetic induction is reduced to solving the Laplace equation using boundary conditions.

In most cases however, the boundary is so complicated that the potential cannot be solved directly. A way to overcome this problem is to transform the complicated boundary to a much simpler one, where the potential is solved much easier. These transformations are performed by analytical functions, which map harmonic functions onto harmonic functions. So the potential problem is solved in two steps: first, perform the geometrical transformation and secondly, solve the Laplace equation in the transformed plane. The transformation function is given by the so called SchwarzChristoffel equation. 
The potential is changed only because of the presence of a coil. A coil is simulated by a linear potential change along the coil boundary.

\section{Results}

We applied the method of $\mathrm{CM}$ to a pole configuration, with a sudden change of the gap. This is shown schematically in fig. 4 . The distance pole face to median plane changes from $g$ to $h$. The $E F B$ for this configuration has already been estimated by Neu and Werner [7]. An expression for $I_{2}$ has been derived by Webers [8]. Using these expressions, we can compare the results from $\mathrm{CM}$ with Poisson calculations and measurements. This is shown in table 1 for $h / g=$ $25 / 19$ and $h / g=25 / 10$. It is seen that, within the measuring error, there is agreement between the measurements and CM. As far as $I_{2}$ is concerned, there is also agreement between the Poisson results and CM. The minor difference in $E F B$ is probably caused by the numerical grid size used by Poisson. The measured magnetic field is less than $0.2 \mathrm{~T}$, so saturation effects can be neglected indeed.

Beside this configuration, we also simulated pole configurations with a coil. Also from these calculations it is seen that there is agreement between the measurements and $\mathrm{CM}$.

Concluding, we can say CM is a useful tool to study the fringe fields of the microtron. Using this description, stability calculations are being done to determine the optimum shape of the magnetic ficld.

\section{The rf accelerating cavity}

The microtron accelerating cavity is a standing wave on axis coupled bi-periodic structure consisting of three accelerating celis and two coupling cells. It operates at $1.3 \mathrm{GHz}$ and gives an energy gain of $2.22 \mathrm{MV}$. Field calculations were carried out with the computer programs SUPERFISH and URMEL-T. In general, the
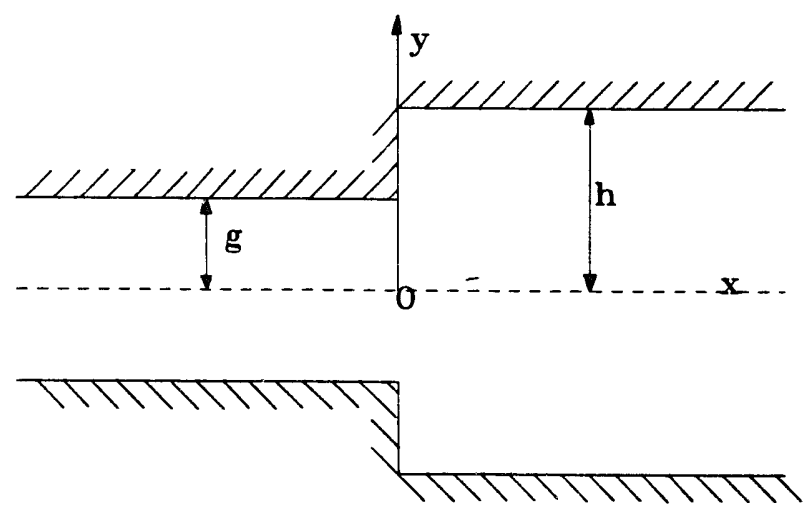

Fig. 4. Configuration with sudden change of gap.
Table 1

$E F B$ and $I_{2}$ for the configuration with a sudden change of the gap

\begin{tabular}{lllll}
\hline & \multicolumn{2}{l}{$h / g=25 / 19$} & \multicolumn{2}{l}{$h / g=25 / 10$} \\
\hline Method & $\begin{array}{l}E F B \\
{[\mathrm{~mm}]}\end{array}$ & $\left|I_{2}\right|$ & $\begin{array}{l}E F B \\
{[\mathrm{~mm}]}\end{array}$ & $\left|I_{2}\right|$ \\
\hline Poisson & 3.7 & 0.38 & 5.2 & 0.50 \\
Measurements & $4 \pm 1$ & $0.35 \pm 0.05$ & $6 \pm 1$ & $0.48 \pm 0.05$ \\
Conformal mapping & 4.8 & 0.37 & 6.2 & 0.50 \\
\hline
\end{tabular}

agreement between these two codes was better than 1\%. Some results are listed in table 2 . The coupling $k$ between neighbouring cells of the cavity was calculated with the 3-dimensional program MAFIA.

In order to understand the influence of small tuning errors in individual cells, we simulated the accelerator structure analytically by an equivalent circuit consisting of a chain of coupled LC resonator cells [9]. For five cells there are five ground modes of which three have even symmetry and two have odd symmetry with respect to the middle of the structure. The coupling factor $k$ and the relative frequency errors of the cou-

Table 2

Accelerating cavity parameters

\begin{tabular}{|c|c|}
\hline Cavity frequency & $1300 \mathrm{MHz}$ \\
\hline Accelerating voltage & $2.22 \mathrm{MV}$ \\
\hline rf wavelength & $23.1 \mathrm{~cm}$ \\
\hline Cavity length & $40 \mathrm{~cm}$ \\
\hline Number of accelerating cells & 3 \\
\hline Number of coupling cells & 2 \\
\hline Maximum beam loading & $94 \%$ \\
\hline $\begin{array}{l}\text { Corr. shunt impedance } \\
\left(V^{2} / P, \text { URMEL-T }\right)(3 \text { cells })\end{array}$ & $21 \mathrm{M} \Omega$ \\
\hline $\begin{array}{l}\text { Macropulse wall losses } \\
\text { (URMEL-T) }(3 \text { cells) }\end{array}$ & $230 \mathrm{~kW}$ \\
\hline $\begin{array}{l}\text { Stored rf energy } \\
\quad(\text { URMEL-T) }(3 \text { cells })\end{array}$ & $0.75 】$ \\
\hline $\begin{array}{l}\text { Transit time factor } \\
\text { (URMEL-T) }\end{array}$ & 0.80 \\
\hline Unloaded $Q$ (MAFIA) & 23500 \\
\hline $\begin{array}{l}\text { Unloaded } Q \\
\quad \text { (measured for Al model) }\end{array}$ & 9400 \\
\hline $\begin{array}{l}\text { First neighbour coupling } k \\
\text { (MAFIA) }\end{array}$ & -0.053 \\
\hline $\begin{array}{l}\text { First neighbour coupling } k \\
\text { (measured) }\end{array}$ & -0.055 \\
\hline $\begin{array}{l}\text { Generator-cavity coupling } \beta \\
\text { (design value) }\end{array}$ & 6 \\
\hline $\begin{array}{l}\text { Generator-cavity coupling } \beta \\
\text { (measured) }\end{array}$ & 2 \\
\hline $\begin{array}{l}\text { Coupling cell frequency error } \\
\text { (measured) }\end{array}$ & $2.0 \%$ \\
\hline $\begin{array}{l}\text { Accelerating cell frequency error } \\
\text { (measured) }\end{array}$ & $0.41 \%$ \\
\hline
\end{tabular}


pling cells $\left(\delta_{\mathrm{c}}\right)$ and the outer two accelerating cells $\left(\delta_{\mathrm{a}}\right)$ with respect to the middle cell can be found from the frequency spectrum of the five modes. The result is [10]

$$
\begin{aligned}
& k=\frac{2}{\sqrt{3}} \frac{\left(\omega_{5}^{2}+\sqrt{3} \omega_{4}^{2}-\sqrt{3} \omega_{2}^{2}-\omega_{1}^{2}\right)}{\omega_{1}^{2}+\omega_{2}^{2}+\omega_{4}^{2}+\omega_{5}^{2}}, \\
& \delta_{\mathrm{a}}=\frac{3\left[\left(\omega_{2}-\omega_{1}\right)-\left(\omega_{5}-\omega_{4}\right)\right]}{2 \omega_{3}}, \\
& \delta_{\mathrm{c}}=\frac{-\omega_{1}+3 \omega_{2}-4 \omega_{3}+3 \omega_{4}-\omega_{5}}{2 \omega_{3}} .
\end{aligned}
$$

An aluminium, scale 1:1 model of the structure was made in order to measure various cavity properties like the generator to cavity coupling coefficient $\beta$ [11], the coupling constant $k$ and the tuning errors $\delta_{\mathrm{a}}$ and $\delta_{\mathrm{c}}$. Field profiles were measured with the perturbation ball method [12]. In fig. 5 we give as an example the measured signal (i.e. resonance frequency shift) for three of the five modes namely the accelerating mode $(\pi / 2$-mode) and the two outer modes ( 0 -mode and $\pi$-mode). We note that the electric field is proportional to the square root of the measured frequency shift.

The accelerating mode shows a too low field in the middle cell. This is caused by tuning errors $\delta_{\mathrm{a}}$ and $\boldsymbol{\delta}_{\mathrm{c}}$.
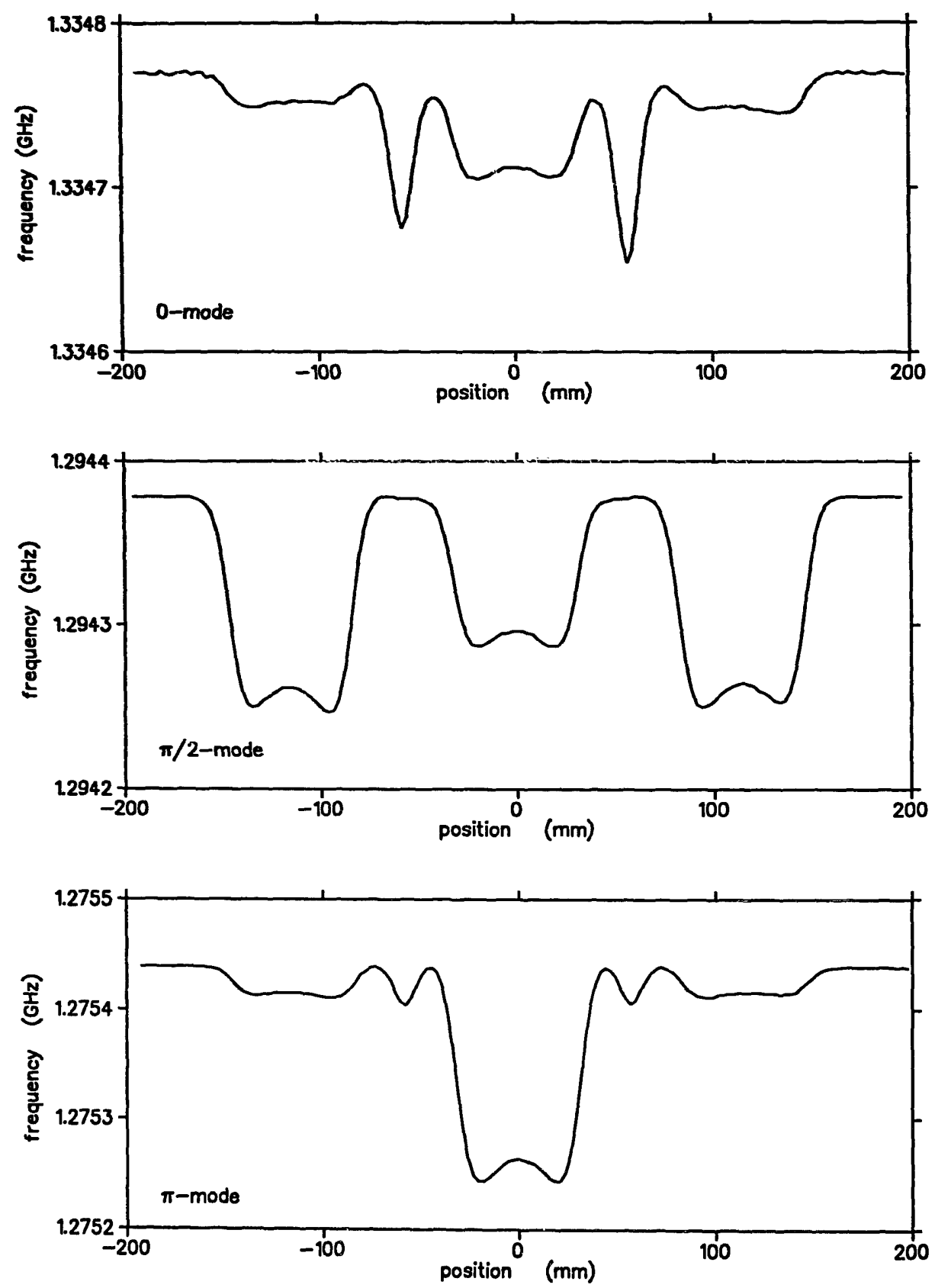

Fig. 5. Field profiles for three of the five ground modes, measured with the perturbation ball method. The electric field is proportional to the square root of the relative frequency shift. 


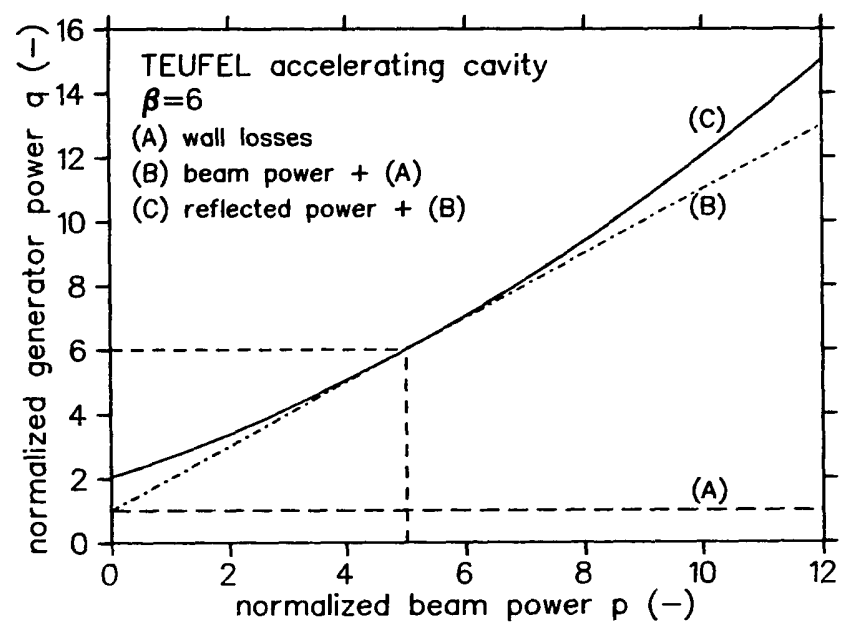

Fig. 6. Required normalized generator power as function of the normalized beam power for $\beta=6$ (normalization w.r.t. the wall losses). The generator power is the sum of the wall losses, the beam power and the reflected power.

In fact, this is a second order effect, but it can still be substantial if the coupling constant $k$ is small. The effect of tuning errors can also be seen by comparing the 0 -mode and the $\pi$-mode. For a perfectly tuned structure the relative field amplitudes in the coupling cells (the narrow peaks) should be equal for these two modes [10]. As can be seen from fig. 5, this is clearly not the case. The measured tuning errors $\delta_{\mathrm{a}}$ and $\delta_{\mathrm{c}}$ are listed in table 2. For the final copper structure these tuning errors will be removed.

At full accelerating current the beam loading for the microtron cavity will be higher than $90 \%$. Therefore, the generator to cavity coupling coefficient $\beta$ should be chosen properly such that for the whole range from zero beam current to its maximum value, the reflected power stays within reasonable limits. For our parameters $\beta=6$ is a reasonable choice [10]. In fig. 6 we give the required generator power as a function of the normalized beam power (i.e. beam power divided by wall losses) for the case $\beta=6$. This result was obtained from an equivalent circuit simulation of the cavity-generator coupling mechanism [11].

The coupling coefficient $\boldsymbol{\beta}$ for the aluminium model cavity was obtained from a measurement of the loaded $Q$-value $\left(Q_{\mathrm{L}}\right)$ and the unloaded $Q$-value $\left(Q_{0}\right)$ by the relation $\beta=\left(Q_{0}-Q_{\mathrm{L}}\right) / Q_{\mathrm{L}}$. We found $\beta=2$ which means that the coupling iris still must be enlarged.

\section{References}

[1] G.J. Ernst, these Proceedings (13th Int. Free Electron Laser Conf., Santa Fe, USA, 1991) Nucl. Instr. and Meth. A318 (1992) 173.

[2] J.I.M. Botman, J.L. Delhez, G.A. Webers, H.L. Hagedoorn, W.J.G.M. Kleeven, C.J. Timmermans, G.J. Ernst, J.W.J. Verschuur, W.J. Witteman and E.H. Haselhoff, Nucl. Instr. and Meth. A304 (1991) 192.

[3] H.R. Froelich, A.S. Thompson, D.S. Edmonds Jr., J.J. Manca, J.W. McGowan, J.C.F. MacDonald, J. Beard and G. Bees, Nucl. Instr. and Meth. 143 (1977) 473.

[4] J.L. Delhez, G.A. Webers, J.I.M. Botman, H.L. Hagedoorn, D. Muzio and C.J. Timmermans, Proc. ECAART2, Nucl. Instr. and Meth. B68 (1991) to appear.

[5] G.A. Webers, J.L. Delhez, J.I.M. Botman, H.L. Hagedoorn, J. Hofman and C.J. Timmermans, Proc. ECAART-2, Nucl. Instr. and Meth. B68 (1991) to appear.

[6] H.A. Enge, Focusing of Charged Particles, ed. A. Septier (Academy Press, New York, 1967).

[7] H. Neu and H. Werner, Nucl. Instr. and Meth. 10 (1960) 333.

[8] G.A. Webers, internal report VDF/NK 91-26, T.U.E.

[9] D.E. Nagle, E.A. Knapp and B.C. Knapp, Rev. Sci. Instr. 38 (1967) 1583.

[10] M.E.H.J. Theeuwen, Eindhoven University of Technology, Cyclotron Laboratory, internal report VDF/NK 9128 (1991).

[11] P.B. Wilson, Physics of High Energy Particle Accelerators, Fermilab Summer School., 1981, ed. R.A. Carrigan, F.R. Huson and M. Month, AIP Conf. Proc. 87 (1982) p. 450.

[12] E. Müller, Diplomarbeit, Goethe-Universität Frankfurt am Main, 1977. 